\title{
Network Simulators Parametric Comparison for Network Mobility Management
}

\author{
Azana Hafizah Mohd Aman ${ }^{1}$, Aisha-Hassan A. Hashim ${ }^{1}$, Azween Abdullah ${ }^{2}$, \\ Huda Adibah Mohd Ramli ${ }^{1}$ and Shayla Islam ${ }^{1}$ \\ ${ }^{1}$ Kulliyyah of Engineering, International Islamic University Malaysia, Jalan \\ Gombak 53100, Kuala Lumpur, Malaysia \\ ${ }^{2}$ SOCIT, Taylor's University, Jalan Taylors, Subang Jaya 47500, Selangor, \\ Malaysia)
}

\begin{abstract}
It is generally known that network simulator is a program used to verify new or existing network architecture and its activities. Basically network simulator helps to implement the virtual part of network research. This has save a lot of cost on analysis as the results of a network simulator is very close to real network cases. In recent researches, new models and prototypes are introduced to enable latest technologies support on network simulators. Some well-known network simulators are NS2, NS3, OMNET++ and QUALNET. As network research continues to grow rapidly, there is a need to analyze the supports and services offered by each network simulators. The objective of this paper is to investigate and analyze these network simulators in term of network mobility supports. This is because network mobility management has become a crucial topic in networking research. In selecting the right network simulators, issues such as CPU utilization, memory usage, computation time, feasibility, scalability and affordability need to be wisely studied. Using qualitative analysis this paper highlights the strengths and the limitations of these network simulators. The qualitative evaluations of these simulators are hereby presented.
\end{abstract}

Keywords: Network Simulator, Simulation, Mobility

\section{Introduction}

Modeling network activities using network simulator give acceptable outputs that are close to real network environments. Network simulators, virtually models and prototypes the behavior of a network. It provides numeric values to enable calculation of the relations between different network entities [1]. The calculations are done using mathematical formulas. It also captures and plays observations from a production network [1]. It supports various attributes of network environments that are modifiable in a controlled manner under different configurations [1]. As the demand for network mobility management activities increase in real network usage, it has become one of the most conferred topics among researcher. Therefore it is necessary to analyses available network simulators that support network mobility management.

This paper investigates and qualitatively analyzes recent available network simulators for network mobility management. The most recent works are deeply analyzed in which an inclusive evaluation is presented. The parameters considered are CPU utilization, memory usage, computation time, feasibility, scalability and affordability.

The paper is organized as follows; section 2 presents the basic of network simulators. Section 3 highlights the selected network simulators. Section 4 discussed the related works. Section 5 describes the qualitative analysis of the selected network simulator. Finally, the conclusion of the paper is presented in Section 6. 


\section{Basic of Network Simulator}

In network research and development, there are three common terms that need to be understood. The three common terms are network simulator, simulations and network emulator [1-2].

A network simulator is a program that foresees the activities of a network. It simplifies the old-fashioned analytical methods to provide an accurate understanding of system behavior of a network. In simulators, the network entities are demonstrated with nodes, devices, links, applications and more. Simulators usually come with support for the most general technologies and networks in use today. It is normally categorized to commercial simulator or open source simulator. The commercial simulators are Graphical User Interface (GUI) driven, while the open source network simulators are Command Line Interface (CLI) driven. The network model and configuration defines the network entities and the events. Example events are data transmissions or packet error. The outputs of these events are recorded as trace files. The trace files log the required packet of the simulation which eventually is used for analysis. Network simulators enable support tools to facilitate visual analysis of trends and potential issues [1-2].

The term simulation is the imitation or testing or running the configured network model in a network simulator. Simulation of networks is a very intricate task. As it is not easy to develop network behavior, such as mobility, multicast, handover, bottleneck or buffer overflows. Estimations normally consider the average occupancy, high variance and the time required for an accurate output. Sampling and control varies are some techniques developed to smooth the simulation [1-2].

A network emulator permits users to host real devices and applications into a virtual test network. It virtually varies packet flow as to imitate the behavior of a real network. Live traffic pass through the simulator and affected objects within the simulation. The usual practice is that real packets from a live application reach the emulation server. The real packet gets modulated into a simulation packet. After experiencing packet loss, handover delay and throughput effects then the simulation packet gets demodulated into real packet. Thus this practice resembles the real packet flow of the real networks even though it flows through the simulated network. Emulation is usually in the design state for confirming communication networks before real deployment [1-2].

Some examples of network simulators or emulators for open source are NS2, NS3, BookSim, SuperSim and Filius [1]. While examples for propriety network simulators are QUALNET, OPNET, OMNET++, NetSim and more [1]. Hence there are many selections of network simulators that range from the very simple to the very complex types. Overall, a network simulator must be able to model and configure network entities and topologies. A network simulator must be able to model and visualize data flows. Other main support is to be able to provide network performance metrics results in numeric values or graphs.

\section{Selected Network Simulators}

This section briefly describes the selected network simulators that supports network mobility management called PMIPv6. There are four selected network simulators namely NS2 [3], NS3 [4], OMNET++ [5] and QUALNET [6].

\subsection{NS2 and NS3}

NS is known as Network Simulator it is a series of discrete event network simulators. Started with NS1, then NS2 and the most recent is NS3. All of them are discrete-event computer network simulators. As open source software it is publicly available online under the GNU GPLv2 license for research, development, and use. The core of NS2 and 
NS3 is written in C++, with Tcl-based scripting of simulation scenarios for NS2 [3-4]. NS3 was built to replace NS2. NS3 used Python bindings (pybindgen) and Waf build system. Both NS2 and NS3 comprised contributions from other researchers, such as wireless code from the UCB Daedelus, CMU Monarch projects and Sun Microsystems [3]. Current statuses of the three versions are NS1 is already absolute, NS2 is no longer actively maintained, while NS3 is aggressively developed.

\subsection{OMNET++}

OMNeT++ is an extensible, modular, component-based C++ simulation library and framework, primarily for building network simulators. Some of its functionalities are sensor networks, wireless ad-hoc networks, Internet protocols, performance modeling, photonic networks, and more. OMNeT++ provides an Eclipse-based IDE, a graphical runtime environment, and a host of other tools. There are extensions for real-time simulation, network emulation, database integration, SystemC integration, and several other functions [5]. OMNeT++ provides component architecture for models in which are programmed in $\mathrm{C}++$, and then assembled into larger components and models using a high-level language (NED). Current status of OMNET++ is still keenly developed.

\subsection{GLOMOSIM/QUALNET}

Global Mobile Information System Simulator (GloMoSim) is network protocol simulation software that simulates wireless and wired network systems. GloMoSim is designed using the parallel discrete event simulation capability provided by Parsec, a parallel programming language. It uses the Parsec compiler to compile the simulation protocols [6]. QualNet is a commercial version of GloMoSim. QualNet simulator consists of planning, testing and training tool that models the behavior of real network activities. QualNet provides a comprehensive environment for designing protocols, creating and animating network scenarios, and analyzing their performance. Current status of GloMoSim/QualNet, is actively industrialized.

\section{Current Development and Implementation}

As there are many network topologies being tested using network simulators, this paper focuses on work done to model PMIPv6 network topology. Basic network model for PMIPv6 is shown in Figure 1. Researches in [7-12], carried the simulation based on network model and signaling call flow as shown in Figure 2 - Figure 7. While the simulations parameters for the simulation in [7-13] are shown in Table 1. 


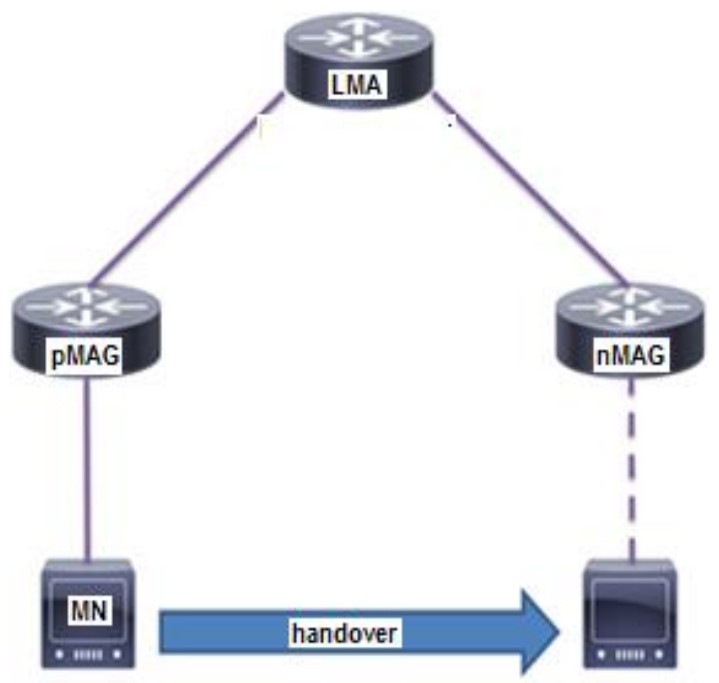

Figure 1. Basic Network Model of PMIPv6

Table 1. Simulation Parameters

\begin{tabular}{|c|c|c|c|c|c|c|c|}
\hline Parameter & \multicolumn{7}{|c|}{ Value } \\
\cline { 2 - 8 } & {$[7]$} & {$[8]$} & {$[9]$} & {$[10]$} & {$[11]$} & {$[12]$} & {$[13]$} \\
\hline Simulator & NS2 & OMNET++ & NS2 & NS3 & NS2 & NS3 & QUALNET \\
\hline No of nodes & 7 & 8 & 6 & 21 & 8 & 8 & 7 \\
\hline $\begin{array}{c}\text { Max Speed } \\
\text { Mbps }\end{array}$ & 50 & 50 & 100 & 100 & 100 & 50 & 54 \\
\hline $\begin{array}{c}\text { Simulations } \\
\text { time }\end{array}$ & $140 \mathrm{~s}$ & $20 \mathrm{~s}$ & $20 \mathrm{~s}$ & $25 \mathrm{~s}$ & $100 \mathrm{~s}$ & $25 \mathrm{~s}$ & $20 \mathrm{~s}$ \\
\hline $\begin{array}{c}\text { Packet size } \\
\text { bytes }\end{array}$ & 1000 & 1000 & 1000 & 1024 & 1000 & 1000 & 100 \\
\hline
\end{tabular}

[7] investigated the performance of PMIPv6 protocol using Network Simulator (NS) version 2.29. It implemented an extension existing MIPv6 to enable PMIPv6 model, configured 6 nodes with 1 mobile node. Making used of the UDP (User Datagram Protocol) and TCP (Transmission Control Protocol) traffics. Performance metrics considered are average handover delay, average packet delivery ratio and throughput. Through simulation experiments value collected are number of packets received, number of packets sent and number of bytes receive. It verified that the proposed architecture and scheme satisfy the flow mobility requirements of PMIPv6. 


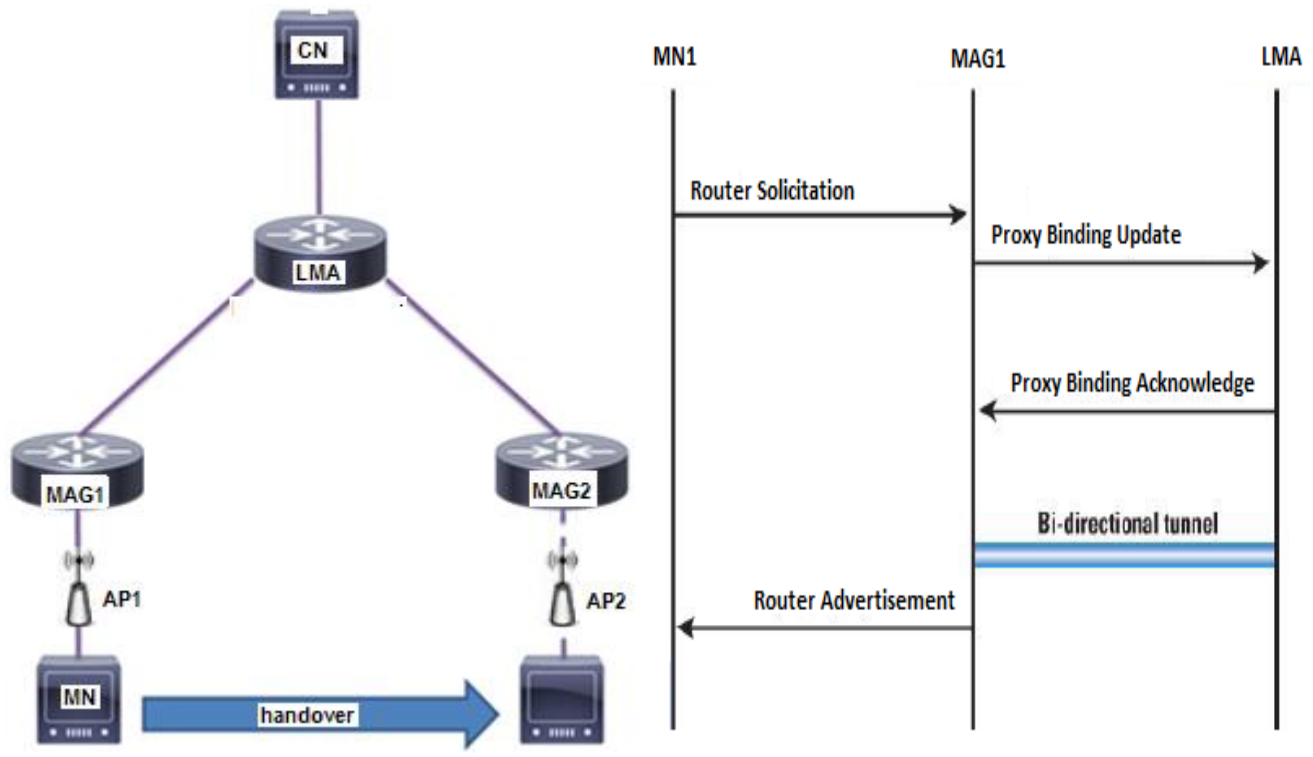

Figure 2. Network Model and Signaling Call Flow for [7]

[8] presented OMNET++ network simulation results for PMIPv6 with Multi-Protocol Label Switching (MPLS). It used xMIPv6 as a base, and modified it to support the MPLS/PMIPv6 protocol. Performance metrics considered are handover delay, handover overhead and end-to-end delay. Simulations are varied by number hop between MAG and LMA. Scenario manager, configurator and channel control modules of OMNET++ are used to support the network model. It verified that the proposed architecture and scheme satisfy the flow mobility requirements of PMIPv6.

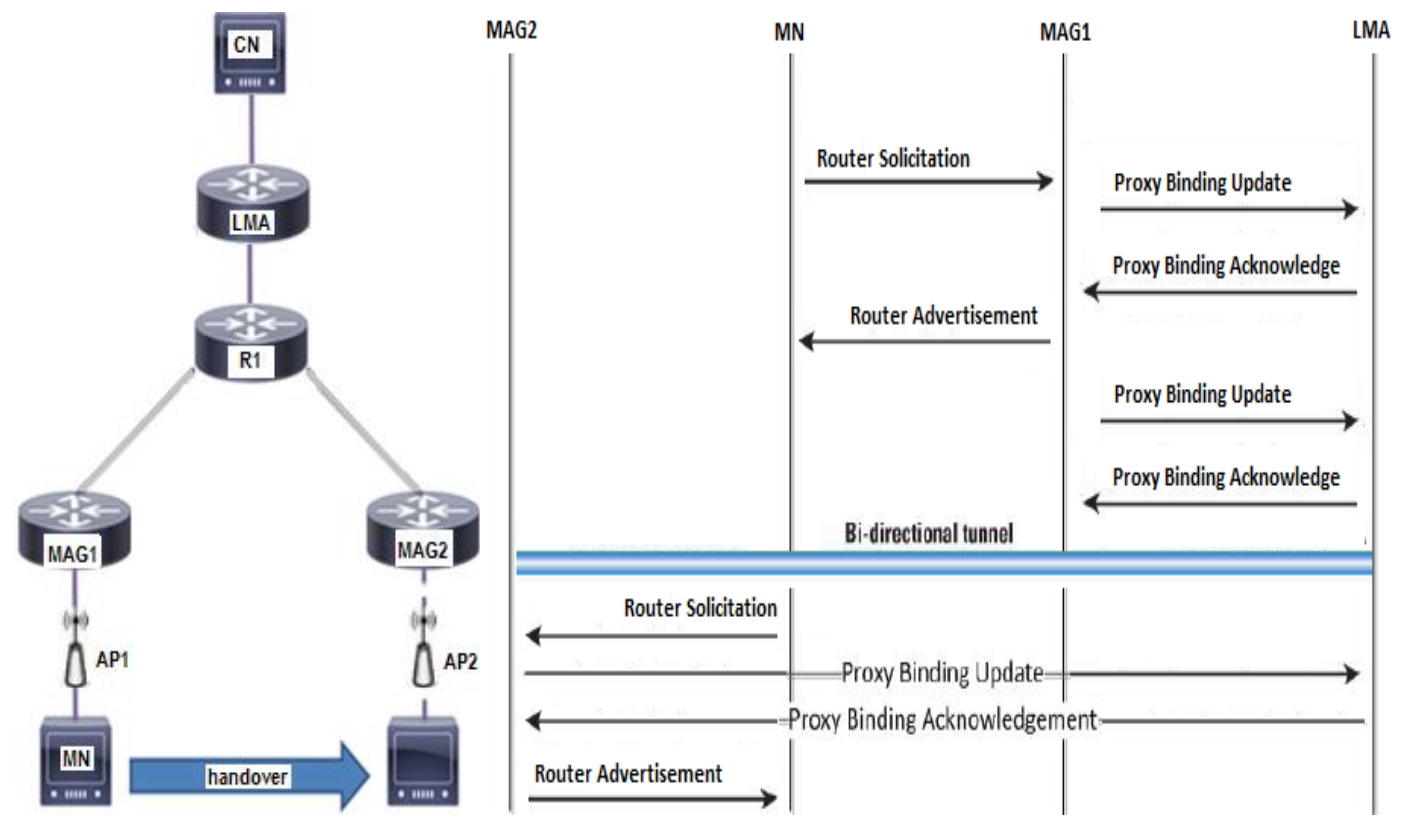

Figure 3. Network Model and Signaling Call Flow for [8] 
[9] implemented authorization, authentication and accounting (AAA) server in PMIPv6 topology. The AAA server and PMIPv6 implementation is via NS-2.29. Performance metric considered are handover and handover delay. Results are calculated based on varying number of LMAs. It verified that the proposed architecture and scheme satisfy the flow mobility requirements of PMIPv6.

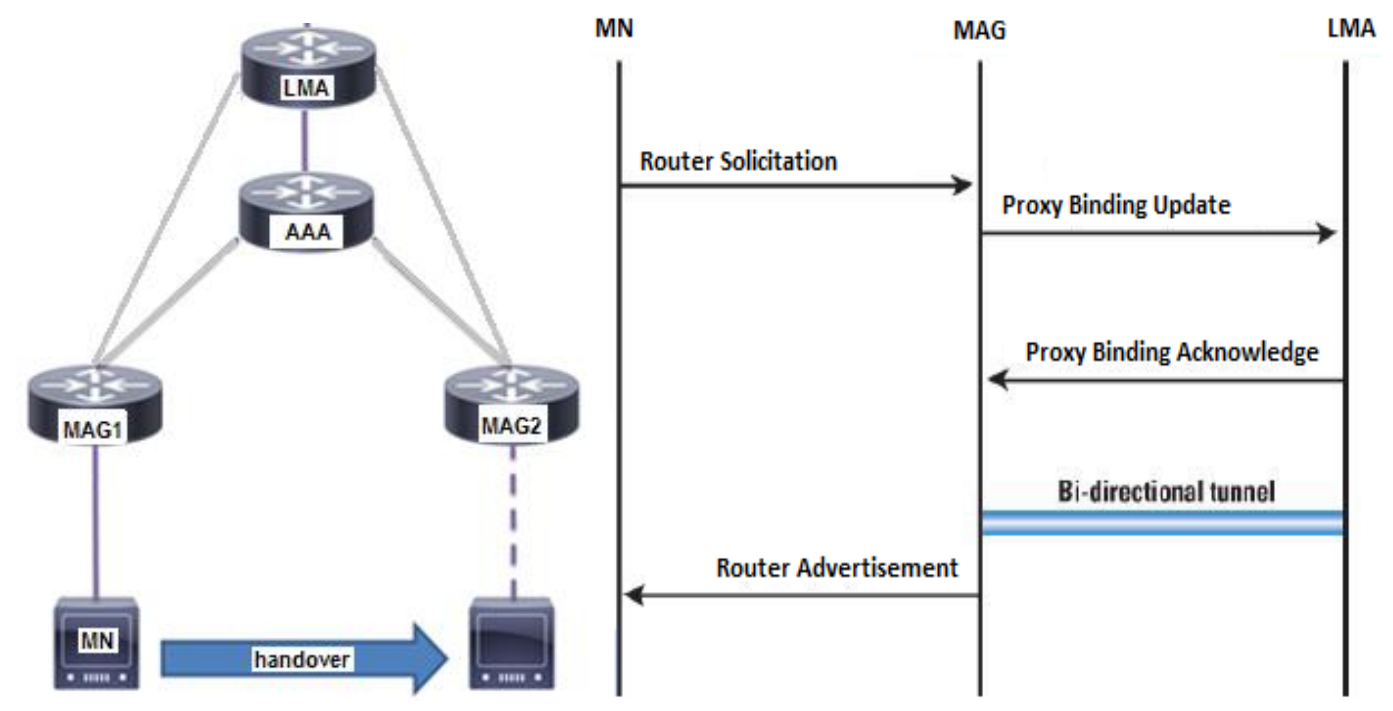

Figure 4. Network Model and Signaling Call Flow for [9]

[10] enhanced flow mobility support using the ns-3 network simulator. By using these ns-3 features, it implemented the multi-interfaced MNs and the flow mobility procedure in the proposed architecture. It verified that the proposed architecture and scheme satisfy the flow mobility requirements. 

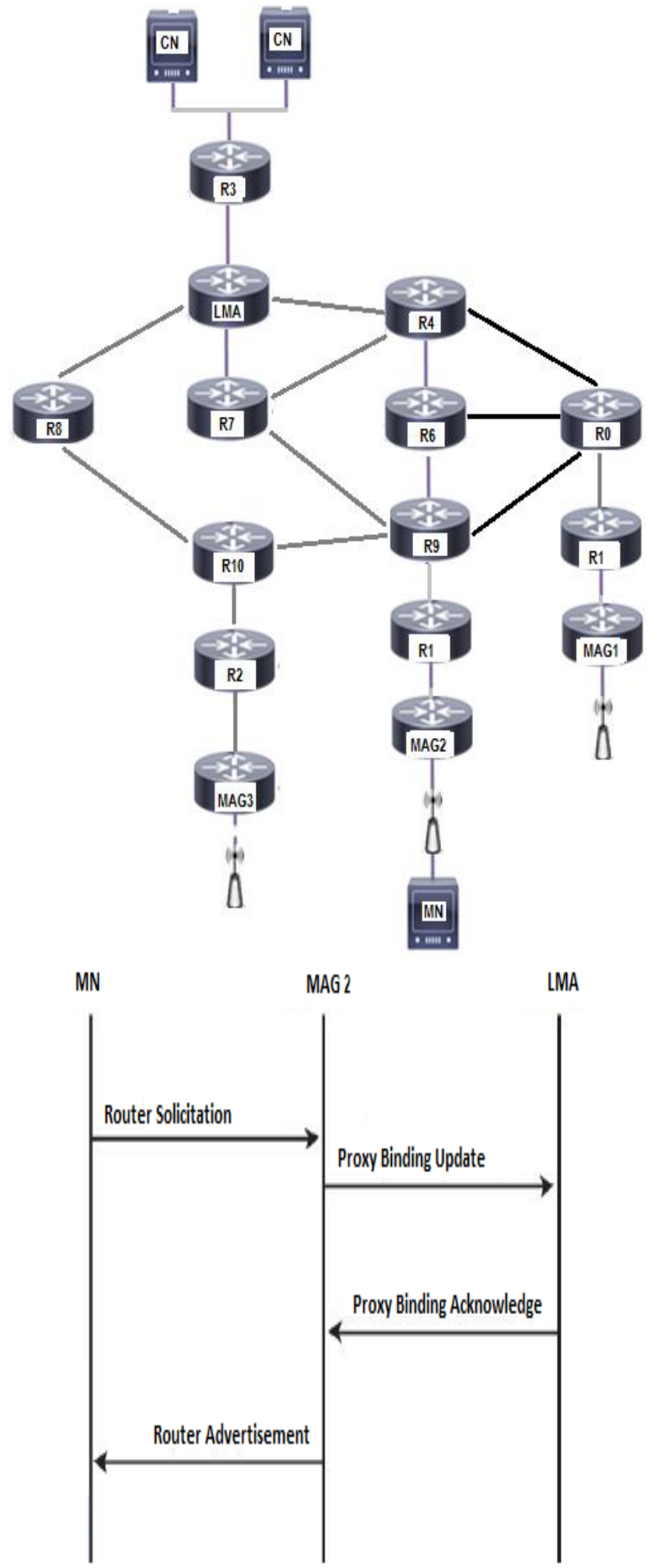

Figure 4. Network Model and Signaling Call Flow for [10]

[11] proposed a secure handover mechanism in PMIPv6 Networks. It implemented group key ticket for fast re-authentication using NS2. Performance metrics considered are handover latency, signaling cost and packet loss. It verified that the proposed architecture and scheme satisfy the flow mobility requirements of PMIPv6. 


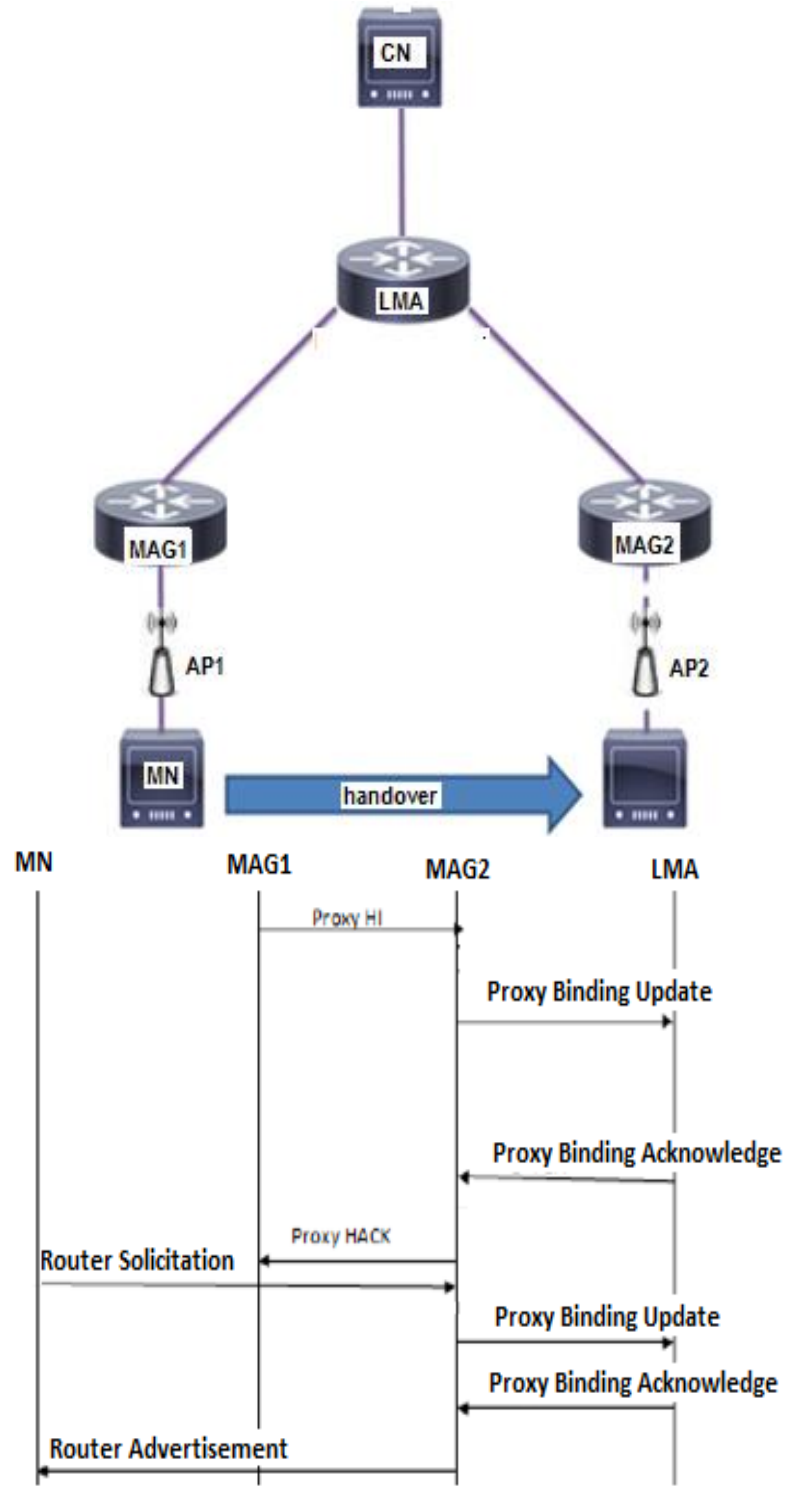

Figure 4. Network Model and Signaling Call Flow for [11]

[12] depicted PMIPv6 model using NS3. It implemented MIPv6 module in NS3 as the base module. Make used of the UDP (User Datagram Protocol) and CBR (Constant Bit Rate Protocol) traffics. Performance metric considered are handover latency and packet drop. The data are collected in a module called PCAP. It verified that the proposed architecture and scheme satisfy the flow mobility requirements of PMIPv6. 


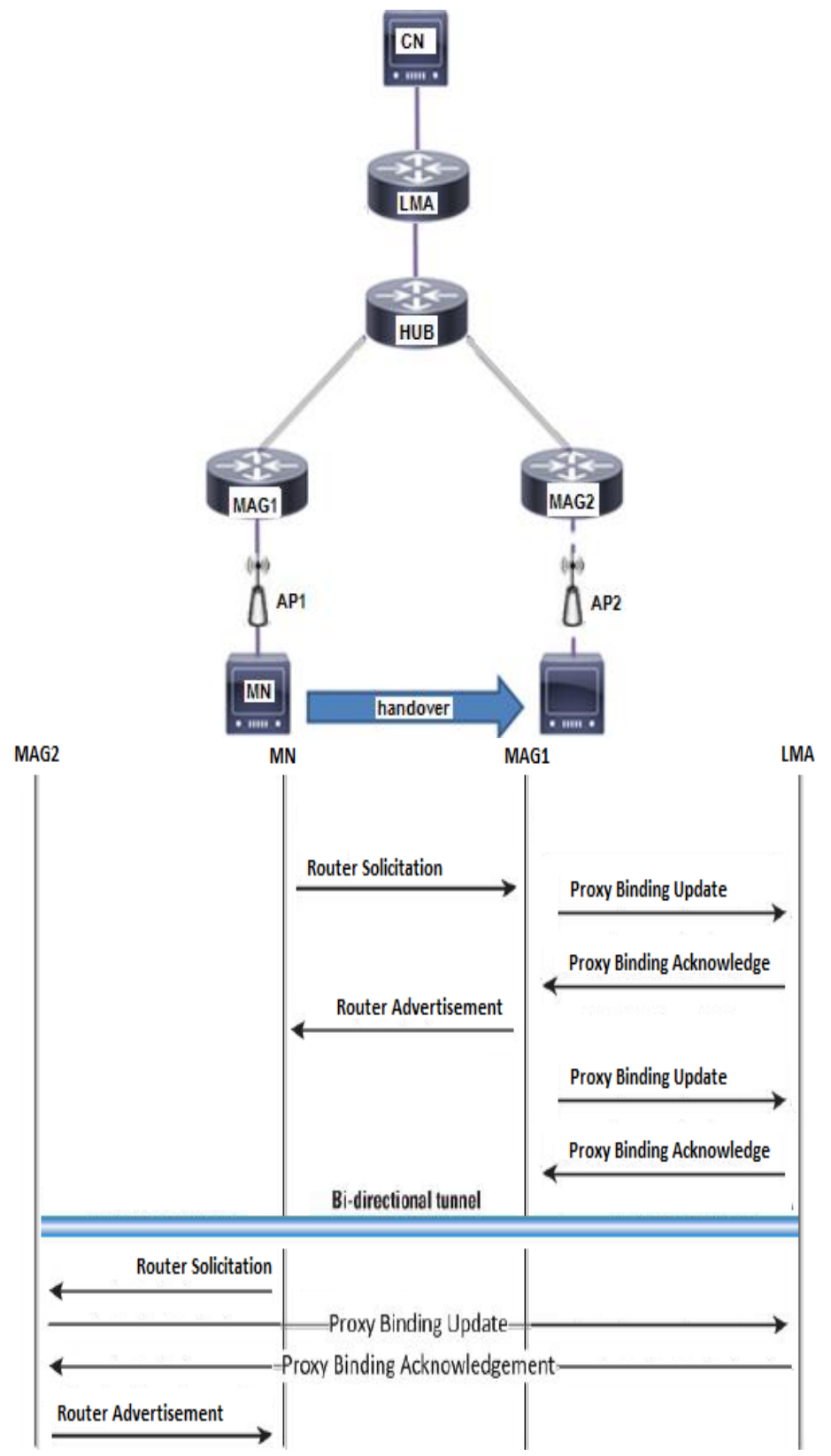

\section{Figure 4. Network Model and Signaling Call Flow for [12]}

[13] investigated the performance of PMIPv6 protocol using QUALNET. In order to apply PMIPv6 module, it implemented two-phase traffic control by extending the queuing discipline of the QUALNET. Performance metrics considered are packet loss and packet buffering. It verified that the proposed architecture and scheme satisfy the flow mobility requirements of PMIPv6. 


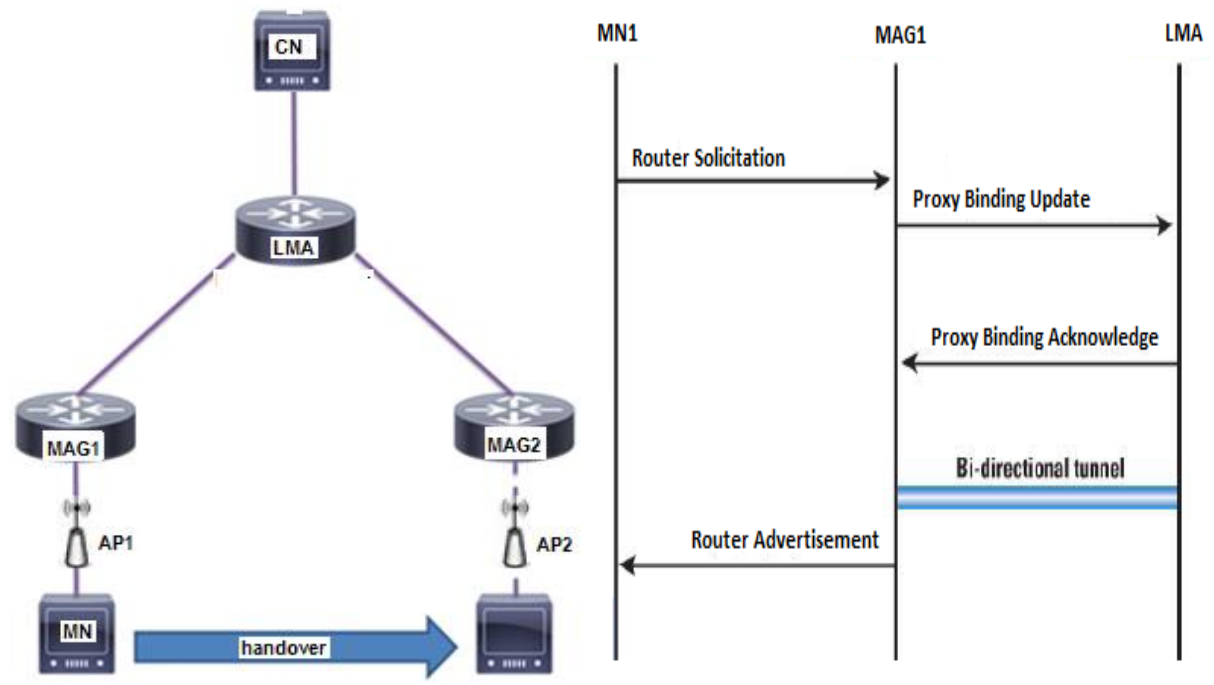

Figure 2. Network Model and Signaling Call Flow for [13]

\section{Qualitative Comparison}

The selected network simulators are generally compared for language, license, platform, tools, feasibility, scalability and ability to interact with the real system. Besides enabling mobility in IPv6, these network simulators must be able to provide network performance metrics results in numeric values. Thus metric such as service disruption time, packet loss rate and handover latency performance issues related to PMIPv6 can be obtained. It is a great bonus if it is able to graphically produce the results.

Table 2 compares the features offered by the selected simulators. By using either NS2, NS3, OMNET++ and QUALNET network simulators the network entities and topology of PMIPv6 is obtained. All the network simulators able to produced numeric values for performance metrics calculations. In terms of licensing, NS2, NS3 and OMNET++ are freely available for academic research, while QUALNET is the most costly. As users are not just using single platform, all network simulators support windows base and linux base operating system. However as for graphical user interface, OMNET++ and QUALNET are the most user friendly. NS2 and NS3 required users to take longer time to be familiar with the command interface. As for scalability NS3 is the most scalable among others.

Table.2. Selected Network Simulators Comparison

\begin{tabular}{|l|c|c|c|c|}
\hline Parameter & Ns2 & Ns3 & Omnet++ & Qualnet \\
\hline Language & C++ & C++ & C++ & Parsec \\
\hline License & Free & Free & $\begin{array}{c}\text { Free (Academic } \\
\text { only)/Commercial }\end{array}$ & Commercial \\
\hline GUI support & No & No & Yes & Yes \\
\hline Platform & $\begin{array}{c}\text { Linux/Windows } \\
\text { based }\end{array}$ & $\begin{array}{c}\text { Linux/Windows } \\
\text { based }\end{array}$ & $\begin{array}{c}\text { Linux/Windows } \\
\text { based }\end{array}$ & $\begin{array}{c}\text { Linux/Windows } \\
\text { based }\end{array}$ \\
\hline Analysis tool & Yes & Yes & Yes & Yes \\
\hline $\begin{array}{l}\text { Visualization } \\
\text { tool }\end{array}$ & Yes & Yes & Yes & Yes \\
\hline Scalability & No & Yes & Yes & Yes \\
\hline Feasability & Yes & Yes & Yes & Yes \\
\hline $\begin{array}{l}\text { Real systems } \\
\text { support }\end{array}$ & Yes & Yes & Yes & Yes \\
\hline
\end{tabular}




\section{Conclusion}

All the selected network simulators are able to provide simulation for PMIPv6 module. However criteria such as affordability and scalability must be considered in academic research. This qualitative analysis only considers criteria for PMIPv6 supports, every network simulators has its own pros and cons depending on the research purpose. Through this study, NS3 is selected as the acceptable PMIPv6 future experimental work because of the scalability and affordability.

\section{Acknowledgements}

This research is funded in part by the Taylors University under grant FRGS/2/2014/ICT01/TAYLOR/03/1

\section{References}

[1] S. Siraj, A. Gupta and R. Badgujar, "Network Simulation Tools Survey, International Journal of Advanced Research in Computer and Communication Engineering", vol. 1, no. 4, (2012)

[2] J. Pan, "A Survey of Network Simulation Tools: Current Status and Future Developments".

[3] NS2 http://www.isi.edu/nsnam/ns/ns-build.html

[4] NS3 https://www.nsnam.org/overview/what-is-ns-3/

[5] OMNET ++ https://omnetpp.org/intro

[6] QUALNET http://web.scalable-networks.com/content/qualnet

[7] F. A. Jalin and R. Alsaqour, "A Simulation Study of Proxy Mobile IPV6 (PMIPV6) Protocol, Journal of Engineering and Applied Sciences", vol. 11, no. 7, (2016).

[8] H. Payappanon, T. Kamolphiwong, K. R. Elz, "Simulation and Evaluation of MPLS based PMIPv6 Network", International J. of Advances in Computer Science and Technology, vol. 2, no. 8, (2013), pp: 07-11.

[9] A. P. Ghatol and N. M. Tarbani, "Optimized Handover Mechanism for Proxy Mobile IPv6", International Journal of Advanced Research in Computer Science and Software Engineering IJARCSE, vol. 5, no. 6, (2015).

[10] H. Y. Choi, S. G. Min, Y. H. Han and R. Koodli, "Design and Simulation of a Flow Mobility Scheme Based on Proxy Mobile IPv6", J Inf Process Syst, vol. 8, no. 4, (2012).

[11] K. Mayuri and K. S. Ranjith, "A Novel Secure Handover Mechanism in Pmipv6 Networks", International Journal of Information Technology Convergence and Services, vol. 4, no. 4, (2014).

[12] H. Y. Choi, S. G. Min, Y. H. Han, J. Park and H. Kim, "Implementation and Evaluation of Proxy Mobile IPv6 in NS-3 Network Simulator", IEEE Xplore, (2011).

[13] T. Usui, "Designing Improved Traffic Control in Network-based Seamless Mobility Management for Wireless LAN", The Third International Conference on Advances in Future Internet, (2011). 
International Journal of Future Generation Communication and Networking Vol. 9, No.10, (2016) 